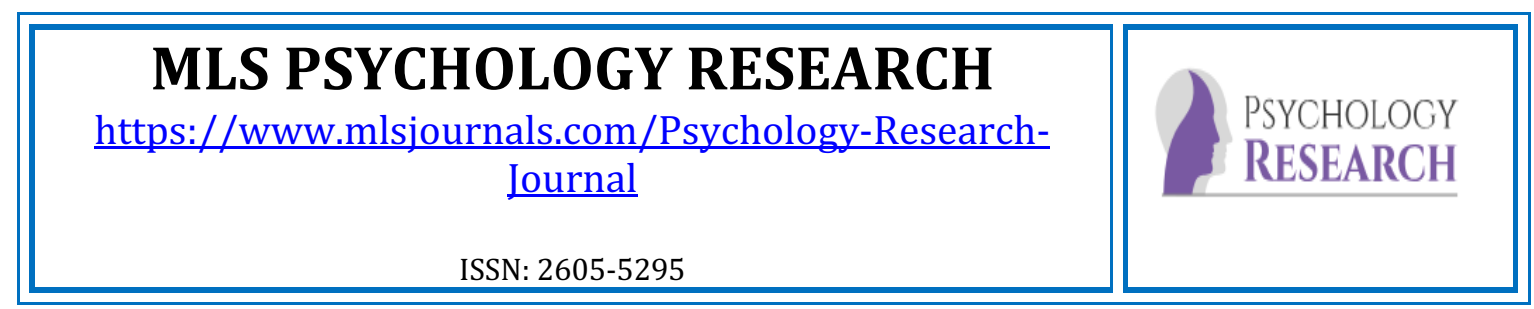

Cómo citar este artículo:

Fierro, A. K. (2019). Percepción del riesgo en trabajos en alturas en empresas de telecomunicaciones de Ecuador y Colombia (agosto-diciembre 2018). MLS Psychology Research 2 (2), 7-22. doi: 10.33000/mlspr.v2i2.289

\title{
PERCEPCIÓN DEL RIESGO EN TRABAJOS EN ALTURAS EN EMPRESAS DE TELECOMUNICACIONES DE ECUADOR Y COLOMBIA (AGOSTO-DICIEMBRE 2018)
}

\author{
Andrea Karina Fierro \\ Movistar (Ecuador) \\ andreafierro77@,hotmail.com
}

\begin{abstract}
Resumen. El artículo está encaminado a presentar de manera científica la correlación existente entre la magnitud del riesgo con relación: a las variables sociodemográficas, a las acciones de prevención que realiza la empresa contratista, al comportamiento del personal y a la percepción del riesgo al ejecutar trabajos en alturas en actividades de operación y mantenimiento de torres de telecomunicaciones. El estudio fue realizado en Ecuador y Colombia. Este tema es de vital importancia por ser una actividad de alto riesgo que debe ser ejecutada con rapidez y precisión debido a la necesidad constante de que el mundo se encuentre comunicado a través de la tecnología. Para obtener los resultados de esta investigación se utilizó un instrumento de medición que consta de 4 bloques de preguntas, con un total de 35 preguntas. El mismo fue aplicado a una muestra que se estableció estadísticamente en 251 trabajadores de empresas proveedoras de servicios de operación y mantenimiento que realizan trabajos en alturas en torres de telecomunicaciones en Ecuador y Colombia. Para el análisis estadístico se utilizó el programa SPSS versión 25. A las respuestas recopiladas se les aplicó el análisis de Kruskall Wallis obteniendo como resultado que cuatro variables influyen en la percepción de la magnitud del riesgo: la gravedad de las consecuencias, el potencial catastrófico, la vulnerabilidad personal y la verificación del estado de los equipos de protección que cada colaborador debe llevar.
\end{abstract}

Palabras clave: Peligro, Percepción del riesgo, Telecomunicaciones, Trabajos en alturas

\section{PERCEPTION OF RISK IN WORK AT HEIGHTS IN TELECOMMUNICATIONS COMPANIES IN ECUADOR AND COLOMBIA (AUGUST-DECEMBER 2018)}

\footnotetext{
Abstract. The article is aimed at presenting in a scientific manner the existing correlation between the magnitude of the risk in relation to the sociodemographic variables, the prevention actions carried out by the contractor, the behavior of the personnel and the perception of risk when executing work at heights in
} 
operations and maintenance of telecommunications towers in Ecuador and Colombia. This issue is of vital importance because it is a high-risk activity, which must be executed quickly and accurately due to the constant need for the world to be communicated through technology. To obtain the results of this research a measurement instrument was used consisting of 4 blocks of questions, with a total of 35 questions. It was applied to a sample that was established statistically in 251 workers of companies providing service operations and maintenance that perform work at heights in telecommunications towers in Ecuador and Colombia. The SPSS version 25 program was used for statistical analysis. The responses collected were the analysis of Kruskall Wallis resulting in four variables influencing the perception of the magnitude of the risk: the severity of the consequences, the catastrophic potential, personal vulnerability and verification of the status of protective equipment that each collaborator must carry.

Keywords: Danger, Perception of risk, Telecommunications, Works in heights

\section{Introducción}

La industria de las telecomunicaciones presentó un desarrollo exponencial en los últimos años. De allí que, en la época actual, es imprescindible estar conectados de manera permanente mediante la tecnología. Debido a la rapidez con la que se requiere poner en funcionamiento la operación celular cuando se presenta algún corte se hace necesario que el servicio se restablezca de manera ágil y oportuna. Por tanto, los trabajadores que ejecutan las tareas de operación y mantenimiento, en torres de telecomunicaciones, deben estar dispuestos y aptos para realizar estas tareas ya que, el trabajo en alturas que involucra trae consigo riesgos inherentes.

El término riesgo es un término utilizado de manera diaria por el ser humano que realiza un esfuerzo preventivo para que no ocurra ninguna situación que conlleve pérdidas materiales o humanas. El riesgo puede ser valorado por un estímulo inducido que es ocasionado por las veces que puede causar pérdidas humanas o materiales, en este caso hablamos de un riesgo objetivo; por otro lado, también existe el riesgo subjetivo, que desde el punto de vista psicosocial tiene un especial interés ya que este tipo de "valoración intuitiva tiene en cuenta tanto el nivel de conocimiento o desconocimiento del peligro como el grado de control que el individuo ejerce sobre él" (Bayés, Portell y Riba 1997, citado por Martínez, Morillejo, Pozo, 2002).

Bajo este contexto podemos decir que para considerar cierta actividad como de riesgo, se debe analizar a las personas como seres cognitivos que buscan información racional. En este ámbito, la piscología busca los motivos que el ser humano tiene para ejecutar alguna acción de peligro, ya sea porque siente una atracción incontrolable o un exagerado optimismo frente a ella.

Por eso es importante estudiar la percepción del riesgo que tienen los trabajadores que realizan trabajos en alturas en tareas de operación y mantenimiento en torres de telecomunicaciones, ya que conllevará a entender, por un lado, las causas del comportamiento y por otro, a establecer medidas preventivas ante la ocurrencia de accidentes de trabajo y enfermedades profesionales.

\section{Marco teórico}

En la revisión literaria que se presenta a continuación, se abordan 5 temas específicos como son el trabajo en alturas, el comportamiento, las actitudes, el riesgo y la percepción del riesgo. 


\section{Trabajo en alturas}

El trabajo en alturas puede ser uno de los más peligrosos que existen precisamente porque puede producir una caída, la cual nace o se deriva del latín casus, que es el participio del verbo 'cadere' (Salvador, 2015).

Este a su vez se relaciona con la raíz indoeuropea Kad (caer), que representa el desplazamiento de un objeto de arriba hacia abajo causada por la acción de su propio peso y por la atracción que sobre él ejerce la tierra.

"El movimiento de caída libre de un cuerpo es un movimiento rectilíneo uniformemente acelerado. Se puede definir caída, pues, como la pérdida de soporte o equilibrio que sufre una persona" (Salvador, 2015, p.1).

Para determinar las lesiones que se pueden producir en una caída, se debe analizar los factores generales como son la altura de la caída, la superficie de impacto, la posición del cuerpo en la caída y otros factores individuales como la edad, el peso del cuerpo, las enfermedades preexistentes y sobre todo la fuerza de gravedad cuyos impulsos aumentan la complejidad de las lesiones (Salvador, 2015).

\section{Comportamiento}

El comportamiento es un proceso físico, "registrable y verificable, que consiste, precisamente, en ser la actividad por la que un ser vivo mantiene y desarrolla su vida en relación con su ambiente, respondiendo a él y modificándolo" (Galarsi, Medina, Ledezma y Zanin, 2011, p.99).

El comportamiento tiene también consigo la "comprensibilidad de la acción y la legalidad de los procesos psico-orgánicos por los que se realiza" (Chauchard, 1961, citado por Galarsi et al., 2011).

El ser humano, mediante la corteza prefrontal planifica un comportamiento cognitivamente complejo, toma decisiones y adecúa su comportamiento social en cada paso, sin embargo, lo más importante que realiza es la conexión entre los pensamientos y las acciones, otorgando la capacidad de impedir comportamientos instintivos (Galarsi et al., 2011).

La conducta humana se encuentra bajo el control del sujeto y nace de un procesamiento de la información disponible que permite tomar decisiones conductuales, pero no siempre la conducta es resultado de un proceso racional o decisional, ya que, en una situación de riesgo, el comportamiento puede ser un reflejo de una acción instintiva o impulsiva (Puyal, s.f.).

Por otro lado, la psicología de la conducta se encamina hacia una posición neutral en el tradicional conflicto del personalismo versus situacionista. "La perspectiva más reciente de la psicología conductual distingue claramente los análisis históricos del individuo, el proceso evolutivo y la síntesis de las distintas experiencias de los determinantes actuales del comportamiento" (Adarraga, Hernández, Márquez y Santacreu, 2002, p. 181).

\section{Actitudes}

"Son esquemas o patrones mentales a partir de los cuales las personas perciben e interpretan el mundo. Estos patrones se configuran con elementos cognitivos (conocimientos) y afectivos (sentimientos), que el individuo va incorporando a lo largo de su vida" (Espulga, 1196, citado por Armengou y López, 2006).

Un estudio realizado por Chisvert, Melià y Pardo (2001), analiza la causalidad de los accidentes de trabajo mediante las explicaciones del por qué ocurren y cómo las 
atribuciones del accidente influyen en las conductas y actitudes hacia la seguridad de los trabajadores.

Existe una compleja relación entre la conducta y la actitud. El determinante inmediato de la conducta es la intención, esta intención conductual está fijada por la actitud frente a la conducta "(evaluación positiva o negativa de la persona de ejecutar esta conducta) y por la norma subjetiva (juicio de la persona de la probabilidad de que otros relevantes, como amigos, compañeros, etc., esperen que él muestre la conducta a pronosticar)" (Chisvert, Meliá y Pardo, 2001, p.6).

\section{Riesgo}

El término riesgo es utilizado de manera diaria por el ser humano que realiza un esfuerzo preventivo para que no ocurra ninguna situación que conlleve a pérdidas materiales o humanas. El riesgo puede ser valorado por un estímulo inducido que es ocasionado por las veces que puede causar pérdidas humanas o materiales, en este caso hablamos de un riesgo objetivo; por otro lado, también existe el riesgo subjetivo, que desde el punto de vista psicosocial tiene un especial interés ya que este tipo de "valoración intuitiva tiene en cuenta tanto el nivel de conocimiento o desconocimiento del peligro como el grado de control que el individuo ejerce sobre él" (Bayés, Portell y Riba 1997, citado por Martínez, Morillejo, Pozo, 2002).

Pero esto es tan solo una división ideológica, ya que para unos "el riesgo es un atributo cuantificable de las tecnologías y peligros de origen natural y aquellos para los que el riesgo es una experiencia subjetiva" (Puy, 1994, p.35), que puede ser pensada, sentida y juzgada.

Existen diferentes tipos de riesgos que pueden afectar a los trabajadores, Díaz (2015) menciona a los siguientes:

- Los originados por las condiciones del centro de trabajo, es decir que dependen de cuan seguras y equipadas sean las instalaciones,

- Los provenientes por agentes físicos, clasificados en: riesgos de tipo mecánico (ruido, vibración), riesgos de tipo calórico o luminoso (iluminación, temperatura), riesgos de tipo de energía (radiaciones, ultra frecuencias),

- Los causados por agentes químicos, que puede ser la exposición a sustancias toxicas, nocivas o corrosivas,

- Los originados por agentes biológicos, entre ellos, virus, bacterias, parásitos, entre otros,

- Los de adaptación al puesto de trabajo, vienen dados por la propia organización, por ejemplo, maquinaria utilizada, pantalla de visualización, silla, mala distribución del trabajo a turnos, entre otros,

- Los de tipo psicológico, se refiere a la influencia del trabajo en la persona, y depende mucho de las características personales del individuo, la sobrecarga de trabajo puede ocasionar, estrés y problemas psíquicos,

- Los riesgos derivados del factor humano, relacionadas con la actitud del trabajador, prácticas inseguras o comportamiento inadecuado en el trabajo.

\section{Percepción del Riesgo}

Los primeros estudios sobre la percepción del riesgo aparecen en los años 60 en la discusión acerca de la energía atómica, sus usos y consecuencias.

De manera posterior, se han realizado varias investigaciones que juegan un papel importante en la toma de decisiones en campos como las ciencias políticas, seguridad industrial, seguridad personal, negocios, desarrollo social, entre otros (Macía, Varela, Vera, 2010). 
Los arranques iniciales de la conceptualización del riesgo fueron de manera predominante de índole técnica y se encontraban ligados a la estadística, con un carácter rigurosamente predictivo (Fischhoff et al., 1978).

En la actualidad con los avances de la filosofía, la tecnología y la globalización, se observa una serie de transiciones en la forma de vivir y descifrar los fenómenos en los cuales nos insertamos, uno de ellos es la noción del riesgo.

Se dice que existen riesgos tangibles y riesgos construidos socialmente a través de pensamientos culturales, y que ningún riesgo es un riesgo per se, por lo que cualesquier situación, condición o circunstancia podría llegar a convertirse en un riesgo para las personas o para la sociedad en general (Macía et al., 2010).

Bajo este contexto podemos decir que para considerar una cierta actividad como de riesgo, se debe analizar a las personas como seres cognitivos que buscan información racional. En este ámbito, la piscología busca los motivos que el ser humano tiene para ejecutar alguna acción de peligro, ya sea porque siente una atracción incontrolable o un exagerado optimismo frente a ella.

"Este optimismo exagerado y obcecado en relación con eventos negativos se denomina ilusión de invulnerabilidad, y el sesgo perceptivo positivo que realiza el individuo respecto a sí mismo y su entorno social se llama optimismo ilusorio" (Blanco, Paez, Rubio, Sanchez, 1998, citado por Martínez et al., 2002).

El mencionar que intervienen variables psicosociales y cognitivas, indica que la percepción del riesgo se encuentra bajo la influencia de patrones culturales, conocimientos previos y factores del medio en que se desenvuelven las personas (González, 2015).

Existen dos modelos basados en un enfoque cognitivo que es necesario señalar ya que son extrapolables a la prevención de accidentes laborables, el "Modelo de Creencias de Salud (MCS) de Maiman y Becker (1974) y la Teoría de la Acción Razonada (TAR) de Fishbein y Azjen (1975)" (Martínez et al., 2002, p. 19).

El modelo MCS, explica la conducta preventiva basada en una amenaza percibida y en una gravedad percibida, que pueden provenir de fuentes internas y externas, como son información previa, accidentes de compañeros, entre otros. Pero lo sobresaliente de este modelo es que "los factores demográficos, de personalidad, estructurales y sociales inciden en la probabilidad de ejecutar la acción saludable a través de las creencias y percepciones subjetivas de las personas" (Martínez et al., 2002, p. 19).

Por su parte, el modelo TAR, considera a las personas como seres racionales y cuya conducta preventiva se basa en la intención determinada por presiones sociales o por creencias de lo que otras personas pueden pensar sobre la realización de la conducta preventiva; este modelo no contempla los factores demográficos ni rasgos de personalidad (Martínez et al., 2012).

Como se puede observar, las dos teorías se basan en lo cognitivo, siendo necesario incluir factores sociales, culturales y sociológicos, como lo es, el rol que desempeña una persona, y que a la hora de entender o actuar con un riesgo tiene influencia, por ejemplo, si es empresario o trabajador.

Bajo este contexto, se propone la necesidad de tener en cuenta un modelo psicosocial acerca de las conductas de riesgo, que logre eliminar las deficiencias de los modelos estrictamente cognitivos, y en el cual se tengan presentes variables psicológico-individuales, así como las variables micro y macrosociales (Martínez et al., 2002, p. 20). 
Por su parte Puy (1994), destaca cuatro enfoques relacionados al estudio de la percepción del riesgo; el enfoque psicológico; el enfoque psicosocial, enfoque cultural y el paradigma psicométrico.

El enfoque psicológico tiene un carácter reduccionista, ya que se basa en enfocar el problema dentro de un ámbito psicológico, individual y cognitivo, es decir que, a la hora de tomar decisiones en condiciones de incertidumbre, las percepciones de las personas son consideradas como errores cognitivos o sesgos.

Esta perspectiva es válida, sin embargo, es criticable "la pretensión de utilizar este tipo de teorías para culpar a las personas por la irracionalidad o el sesgo de sus respuestas ante determinados riesgos, ignorando otros factores de carácter más psicosocial, sociológico y cultural" (Puy, 1994, p.81).

El enfoque psicosocial se basa en la precepción del riesgo como una actitud de la persona y sus respuestas están relacionadas con creencias, valores y variables sociodemográficas.

El enfoque sociológico y cultural, busca respuestas más humanas con relación al riesgo, las mismas que son influenciadas de manera directa por las creencias, actitudes y valores de un grupo de personas que buscan evitar aspectos que puedan poner en peligro su relación con la sociedad (Puy, 1994).

El enfoque psicométrico se refiere a cómo las personas perciben los riesgos de distintas fuentes considerando atributos cualitativos "que inciden en el riesgo percibido más que en la mera probabilidad objetiva de provocar daños o muerte" (Puy, 1994, p.91).

En resumen, la percepción del riesgo es un factor que determina la conducta "ante situaciones potencialmente peligrosas en los múltiples contextos en los que está inmerso el individuo, especialmente en lo respecta al ámbito laboral "(Martínez et al., 2002, p. 18).

En lo que se refiere a estudios específicos, en el año 2002, se presenta un artículo denominado "Percepción de riesgo: Una aproximación psicosocial al ámbito laboral", en el cual se manifiesta que de manera adicional a la percepción del riego que tiene cada individuo, es necesario considerar los factores psicosociales, actitudes de la persona, normas a seguir, presión grupal, entre otros, que pueden modificar las percepciones acerca del riesgo (Martínez et al., 2002).

Así mismo en el año 2010 se realizó una investigación de la percepción del riesgo y salud ocupacional enfocada desde los paradigmas del riesgo (Macía et al., 2010).

En el año 2012, se publica el trabajo llamado "Determinación de riesgo ocupacional en población laboral con trabajo en altura por medición sérica de marcadores", determinando con un enfoque médico los riesgos que implica el trabajo en alturas de acuerdo con el estado de salud del trabajador (Lozano y Mazenett, 2012).

Por otro lado, en el año 2015, se realizó un estudio de los accidentes de trabajo en alturas en el sector agrícola, que permitió comprender las consecuencias de estos, analizar las lesiones y resaltar los peligros que existen en este sector [15]. En ese mismo año, se publica el resumen del trabajo llamado "Risk assessment of work accidents during the installation and maintenance of telecommunication networks" sin que sea posible obtener la información detallada del mismo (Dragoi, Pavalouis, Rosu (2015)

Lo más reciente, es la investigación realizada en el año 2017 a los conductores de bus de una empresa de transporte público en Colombia, en donde se pudo relacionar los accidentes de tránsito ocurridos en la empresa con las conductas inseguras de los trabajadores determinadas en el estudio (Torres, 2017). 
A través del estado del arte, se ha encontrado evidencia de estudios realizados sobre el comportamiento de los trabajadores analizando la percepción que tienen sobre el riesgo, de la misma manera existen estudios relacionados con los trabajos en alturas que se realizan en varias industrias.

\section{Método}

El estudio corresponde a una investigación no experimental transeccional o también llamada transversal, que incluye los exploratorios, descriptivos $\mathrm{y}$ correlacionales-causales, los cuales han sido aplicados en esta investigación (Hernández et al., 2014)

Se inició el estudio aplicando la concepción exploratoria, que permitió conocer la situación actual de las empresas que brindan servicios de operación y mantenimiento para empresas de telecomunicaciones en Ecuador y Colombia, en relación con el monitoreo y seguimiento de los procedimientos de trabajo.

Posteriormente, mediante un enfoque descriptivo se identificó el comportamiento de los trabajadores y su percepción con relación al riesgo de trabajo en alturas en torres de telecomunicaciones, aplicando un instrumento de investigación tipo encuesta que fue previamente validado por expertos y que utiliza la escala de Likert del uno al cinco, con excepción de la última pregunta que utiliza una escala de cero a 100 en intervalos de cinco.

El cuestionario constó de cuatro bloques, el primero corresponde a variables sociodemográficas, descritas en la tabla 1.

Tabla 1

Variables sociodemográficas

Variables

S1

S2

S3

S4

S5

S6

\section{Descripción}

Edad

País

Cargo

Género

Estado Civil

Número de hijos

Nota: Fuente: Elaboración propia, 2019

El segundo bloque corresponde a variables relacionadas a las actividades de prevención que ejecuta la empresa en la cual trabaja el colaborador y se encuentran representadas en la tabla 2 . 
Tabla 2

Variables asociadas a las actividades de prevención de la empresa proveedora

Variables

Descripción

A1 de protección que necesita para realizar trabajos en alturas?

A2 ¿Recibe capacitación sobre trabajos en alturas?

¿La empresa realiza la verificación del estado de los equipos de

A3 protección personal que le asignaron? previo de consumo de drogas o alcohol a cargo de su empleador?

$¿$ Recibe por parte de su empresa un permiso para trabajos en alturas

A5 antes de realizar la tarea?

Nota: Fuente: Elaboración propia, 2019, basada en Rodriguez et al (2013).

El tercer bloque representado en la tabla 3, corresponde a variables de comportamiento personal, es decir acciones que realizan los trabajadores antes y durante el trabajo en alturas.

Tabla 3

Variables asociadas a comportamiento personal

Variables

B1

B2

B3

B4

B5

B6

\section{Descripción}

¿Está familiarizado con los procedimientos que debe realizar para ejecutar trabajos en alturas?

¿Sube a realizar trabajos en alturas llevando consigo su teléfono celular o tablet?

¿Realiza una revisión de los equipos que debe llevar cuando va a realizar trabajos en alturas?

¿Verifica que los equipos de protección personal se encuentren en buen estado antes de realizar trabajos en alturas?

¿Cree que para que ocurra un accidente influye el estado de salud de la persona?

¿Le parece divertido fumar o comer cuando realiza trabajos en alturas?

Nota: Fuente: Elaboración propia, 2019, basada en Rodriguez et al (2013).

El cuarto bloque representado en la tabla 4, corresponde a variables de riesgo percibido, así como el factor de riesgo explorado para cada una de ellas. 
Tabla 4

Variables asociadas al riesgo percibido

\begin{tabular}{|c|c|c|}
\hline Variables & Descripción & Factor Explorado \\
\hline $\mathrm{C} 1$ & $\begin{array}{l}\text { ¿Conoce el riesgo asociado al trabajo en } \\
\text { alturas que realiza? }\end{array}$ & $\begin{array}{l}\text { Conocimiento del propio } \\
\text { trabajador }\end{array}$ \\
\hline $\mathrm{C} 2$ & $\begin{array}{l}\text { ¿Considera que los responsables de la } \\
\text { prevención en su empresa conocen el riesgo } \\
\text { asociado al trabajo en alturas? }\end{array}$ & $\begin{array}{l}\text { Conocimiento del } \\
\text { Responsable de Seguridad } \\
\text { y Salud }\end{array}$ \\
\hline $\mathrm{C} 3$ & $\begin{array}{l}\text { ¿Cuánto teme al daño que le pueda ocurrir } \\
\text { mientras realiza trabajos en alturas? } \\
\text { ¿Qué posibilidad tiene usted de experimentar } \\
\text { un daño como consecuencia de la realización }\end{array}$ & Temor \\
\hline $\mathrm{C} 4$ & $\begin{array}{l}\text { de su trabajo? } \\
\text { ¿Qué posibilidad tiene de evitar que esta }\end{array}$ & Vulnerabilidad personal \\
\hline $\mathrm{C} 5$ & $\begin{array}{l}\text { actividad desencadene una situación de } \\
\text { riesgo? }\end{array}$ & $\begin{array}{l}\text { Acción preventiva (control } \\
\text { fatalidad) }\end{array}$ \\
\hline C6 & $\begin{array}{l}\text { En una situación de riesgo que pueda } \\
\text { producirse al realizar trabajos en alturas, ¿qué } \\
\text { posibilidad tiene de intervenir para } \\
\text { controlarla? } \\
\text { ¿Es posible que se puedan producir } \\
\text { situaciones de riesgo en las que se vean } \\
\text { afectadas más de una persona al ejecutar }\end{array}$ & $\begin{array}{l}\text { Acción protectiva (control } \\
\text { del daño) }\end{array}$ \\
\hline $\mathrm{C} 7$ & $\begin{array}{l}\text { trabajos en alturas? } \\
\text { ¿En caso de producirse una situación de }\end{array}$ & Potencial catastrófico \\
\hline $\mathrm{C} 8$ & $\begin{array}{l}\text { riesgo al realizar trabajos en alturas, la } \\
\text { gravedad del daño que le puede causar es? } \\
\text { ¿Cuándo considera que pueden aparecer }\end{array}$ & $\begin{array}{l}\text { Gravedad de las } \\
\text { consecuencias }\end{array}$ \\
\hline C9 & $\begin{array}{l}\text { problemas de salud relacionados con el } \\
\text { trabajo en alturas? }\end{array}$ & $\begin{array}{l}\text { Demora de las } \\
\text { consecuencias }\end{array}$ \\
\hline
\end{tabular}

Nota: Fuente: Elaboración propia, 2019, basada en basada en Rodriguez et al (2013), Fischhoff et al. (1978).

Utilizando las variables de las tablas 1, 2, 3 y 4, se efectuó un análisis estadístico correlacional en el programa SPSS 25 con la variable (D1) "magnitud del riesgo" obtenida en la última pregunta de la encuesta.

La población que se consideró para este estudio fue de 216 trabajadores en Ecuador y 500 trabajadores en Colombia, es decir la población total fue de 716 trabajadores.

Para establecer el tamaño de la muestra se utilizó el muestreo aleatorio simple, determinando que, para una población de 716 trabajadores, se requiere aplicar el instrumento de investigación a 251, sin embargo, se lo aplicó a 273 trabajadores para prevenir los datos perdidos. Esta encuesta fue realizada entre agosto y diciembre del 2018. Para el caso de Ecuador, respondieron a la encuesta de manera física 62 trabajadores y en Colombia respondieron de manera digital 211 trabajadores.

\section{Resultados}


Para este estudio, las respuestas obtenidas demuestran que el $88 \%$ de los encuestados tienen más de tres años realizando trabajos en alturas; el $88 \%$ de los encuestados recibe cuatro o más capacitaciones de trabajos en alturas al año y el $69 \%$ ha completado los estudios de tercer nivel, con lo cual la muestra refleja que los trabajadores son personal con amplia experiencia, capacitación y preparación para ejecutar trabajos en alturas de operación y mantenimiento en torres de telecomunicaciones.

Con la finalidad de demostrar si existe algún tipo de correlación entre las variables sociodemográficas de la tabla 1 con la variable D1 "magnitud del riesgo", Se sometió a esta variable (D1) a una prueba de normalidad, mediante el ensayo de Anderson Darling y Kolmogorov- Smirnov, en el cual se comprobó, que la variable no presenta una distribución normal, por lo cual, para realizar las correlaciones se utilizó el análisis estadístico denominado Kruskall Wallis para datos no normales, el mismo que representa la alternativa no paramétrica al test ANOVA y que se refleja en la tabla 5.

Tabla 5

Comparación entre magnitud del riesgo y variables sociodemográficas

\begin{tabular}{ll}
\hline Variables & P \\
\hline S1 & .867 \\
S2 & .070 \\
S3 & .713 \\
S4 & .115 \\
S5 & .709 \\
S6 & .129 \\
\hline
\end{tabular}

Nota: Valor de referencia de Pvalue $<.05$, la diferencia entre las medianas no es estadísticamente significativa.

Fuente: Elaboración propia, 2019

En la tabla 5 se observa, que la variable S4 presenta un valor de P Value menor a .05 , por lo tanto su mediana es estadísticamente significativa en relación a la variable D1 "magnitud del riesgo". Para todas las demás variables no existe significancia.

En la tabla 6 se muestra los resultados de la medida de tendencia central llamada media aritmética, las medidas de dispersión: varianza y desviación típica y finalmente la correlación entre la variable D1 "magnitud de riesgo", con las variables asociadas a las actividades de prevención que realiza la empresa en la que el colaborador trabaja y que fueron señaladas en la tabla 2. 
Comparación entre magnitud del riesgo y acciones de prevención que realiza la empresa proveedora.

\begin{tabular}{ccccc}
\hline Variables & $\mathbf{P}$ & Media & $\boldsymbol{\sigma}^{\mathbf{2}}$ & $\boldsymbol{\sigma}$ \\
\hline A1 & .509 & 4,392 & .775 & .979 \\
A2 & .19 & 3,839 & 1,517 & 1,226 \\
A3 & .18 & 2,586 & 2,732 & 1,600 \\
A4 & .821 & 4,143 & 1,231 & 1,259 \\
A5 & .297 & 4,505 & .345 & .928 \\
\hline
\end{tabular}

Valor de referencia Pvalue $<.05$, la diferencia entre las medianas no es estadísticamente significativa. $\sigma^{2}=$ Varianza

Fuente: Elaboración propia, 2019

El P value obtenido en la tabla 6 demuestra que las medianas de las variables de acciones que realiza la empresa para prevenir riesgos en la ejecución de trabajos en alturas de la tabla 2, no son significativas en relación con la variable D1 "magnitud del riesgo".

Por su parte, el análisis de la media aritmética demuestra que únicamente la variable A3, que hace referencia a si la empresa realiza la verificación de los equipos de protección personal, se encuentra en un valor por debajo de tres, lo que indica que los trabajadores perciben que el responsable de la empresa no siempre realiza esta acción. De igual manera, la varianza al presentar un valor alto para la variable A3, demuestra una elevada dispersión de los valores obtenidos.

Continuando con los resultados, en la tabla 7, se presenta la comparación entre la percepción del riesgo y las variables de comportamiento personal señaladas en la tabla 3 y que hacen referencia a las acciones que realiza el colaborador previo a su trabajo en alturas en torres de telecomunicaciones.

Tabla 7

Comparación entre magnitud del riesgo y comportamiento personal

\begin{tabular}{ccccc}
\hline Variables & $\mathrm{P}$ & Media & $\sigma^{2}$ & $\sigma$ \\
\hline B1 & .868 & 4,784 & .236 & .486 \\
B2 & .178 & 4,341 & 1.107 & 1,053 \\
B3 & .129 & 4,780 & .326 & .571 \\
B4 & .013 & 4,813 & .270 & .519 \\
B5 & .818 & 4,527 & .706 & .840 \\
B6 & .131 & 1,300 & .659 & .812 \\
\hline
\end{tabular}

Valor de referencia Pvalue $<.05$, la diferencia entre las medianas no es estadísticamente significativa. $\sigma^{2}=$ Varianza; $\sigma=$ Desviación típica

Fuente: Elaboración propia, 2019

El resultado de la tabla 7 muestra que la mediana de la variable (B4) “ ¿Verifica que los equipos de protección personal se encuentren en buen estado antes de realizar trabajos en alturas?", es significativa en relación con la variable D1 "magnitud del riesgo". Es decir que los trabajadores consideran importante verificar el estado de los EPPS antes de ejecutar trabajos en alturas en torres de telecomunicaciones.

Por su parte, el análisis de la media aritmética demuestra que la variable B6, que hace referencia a si le parece divertido fumar o comer cuando realiza trabajos en alturas, se encuentra en un valor por debajo de dos, lo que indica que a los trabajadores no les parece divertido hacerlo. Los resultados de la varianza demuestran una ligera dispersión 
en los datos obtenidos en la variable B2 que hace referencia a si el colaborador sube a realizar trabajos en alturas llevando consigo su teléfono celular o tablet.

El análisis realizado en la tabla 8 indica la comparación entre la variable D1 "magnitud del riesgo" con las variables asociadas al riesgo percibido, señaladas en la tabla 4.

Tabla 8

Comparación entre la magnitud del riesgo con el riesgo percibido

\begin{tabular}{ccccc}
\hline Variables & $\mathrm{P}$ & Media & $\sigma^{2}$ & $\sigma$ \\
\hline C1 & .295 & 4,788 & .64 & .513 \\
C2 & .420 & 4,275 & .891 & .944 \\
C3 & .005 & 4,396 & .961 & .980 \\
C4 & .005 & 3,817 & 1.613 & 1.27 \\
C5 & .191 & 4,267 & .932 & .965 \\
C6 & .413 & 4,077 & .931 & .965 \\
C7 & .006 & 4,051 & 1.151 & 1.073 \\
C8 & .000 & 4,586 & .640 & .800 \\
C9 & .359 & 2,813 & 1.561 & 1.250 \\
\hline
\end{tabular}

Nota: Valor de referencia Pvalue $<.05$, la diferencia entre las medianas no es estadísticamente significativa. $\sigma^{2}=$ Varianza $\sigma=$ Desviación típica

Fuente: Elaboración propia, 2019

El resultado de la tabla 8 demuestra que las medianas de dos variables de la tabla 4 son significativas en relación con la magnitud del riesgo (D1). Las variables son: (C7) que consulta si es posible que se puedan producir situaciones de riesgo en las que se vean afectadas más de una persona al ejecutar trabajos en alturas, es decir que la magnitud del riesgo tiene relación con el factor denominado potencial catastrófico.

Finalmente, la variable (C8) que consultaba ¿En caso de producirse una situación de riesgo al realizar trabajos en alturas, la gravedad del daño que le puede causar es? Este resultado muestra la relación de la magnitud del riesgo con el factor denominado gravedad de las consecuencias.

El análisis de la media aritmética presenta un valor por debajo de tres para la variable (C9) que se refiere a ¿cuándo considera que pueden aparecer problemas de salud relacionados con el trabajo en alturas?, es decir que los trabajadores piensan que una enfermedad a causa del trabajo no aparece de manera inmediata sino a largo plazo.

$\mathrm{La}$ varianza presenta valores dispersos en las variables $\mathrm{C} 4$ "posibilidad de experimentar un daño"; C7 "posibilidad de producirse situaciones de riesgo en que se vean afectadas más de una persona y C9 "cuando considera que aparecen enfermedades a causa del trabajo".

\section{Discusión y conclusiones}

El estudio realizado aporta con información importante sobre la percepción del riesgo que tienen los trabajadores que realizan tareas de operación y mantenimiento de torres de telecomunicaciones cuando ejecutan trabajos en alturas.

El estudio fue realizado en Ecuador y Colombia, por ser países latinoamericanos con variables económicas, socioculturales y legislación laboral similar, además de la proximidad geográfica, lo cual facilitó la recolección de información y la comparación de resultados bajo un contexto análogo, concluyendo de manera inicial que no existe 
diferencia entre la percepción del riesgo al ejecutar trabajos en alturas en los colaboradores de ambos países.

En relación con el nivel de estudios, el $69 \%$ de los trabajadores encuestados han completado sus estudios de tercer o cuarto nivel, un $28 \%$ tienen estudios de bachillerato y únicamente el 3\% cuenta con un nivel de educación básica, con lo cual podemos decir que la muestra refleja que los trabajadores encuestados son personal con conocimientos técnicos importantes para la ejecución de la tarea.

En lo que se refiere a capacitación, el $94 \%$ de los encuestados asegura que recibe una o más capacitaciones de trabajos en alturas al año y solo el $6 \%$ asegura que durante el año no recibe capacitaciones, lo que permite concluir que las empresas proveedoras de servicio de operación y mantenimiento se preocupan porque su personal se encuentre formado y entrenado en conocimientos y habilidades al ejecutar trabajos en alturas.

En relación con la experiencia en realizar las tareas de operación y mantenimiento en torres de telecomunicaciones, los resultados de la encuesta muestran que el $88 \%$ de los encuestados tienen más de tres años realizando este tipo de trabajos, lo que significa que, en la muestra analizada, los colaboradores cuentan con amplia experiencia ejecutando trabajos en alturas.

En analogía con el cargo que ocupan, entre los colaboradores que completaron la encuesta, se encuentra que el $73 \%$ son trabajadores de altura (torreros), es decir los que propiamente ejecutan el trabajo en la torre, en tanto que el $27 \%$ corresponde a jefes, quienes supervisan las tareas de los torreros. Con esto se puede asegurar que los resultados corresponden a la percepción que tienen los trabajadores que realizan estas tareas de manera permanente.

En el caso de accidentes ocurridos realizando trabajos en alturas, únicamente el 6\% de los encuestados asegura que ha sufrido alguna vez un accidente de trabajo, si se calculó que el $73 \%$ son torreros, de estos únicamente el 3\% ha sufrido alguna vez un accidente, por lo tanto, se puede asegurar que el personal se encuentra altamente capacitado, tiene experiencia y sobre todo tiene cautela al ejecutar trabajos en alturas.

De los trabajadores que han sufrido accidentes de trabajo, todos aseguran que han recibido al menos una capacitación en alturas al año, así también, todos llevan entre $1 \mathrm{o}$ más años de experiencia con lo que se comprueba que los accidentes son sucesos repentinos que pueden ocurrir en cualquier momento y a cualquier persona.

El 96\% de los trabajadores aseguran que el riesgo de ejecutar trabajos en altura en torres de telecomunicaciones es alto; el $85 \%$ le temen al daño y el $67 \%$ piensan que pueden llegar a experimentar un daño a consecuencia del trabajo.

El 75\% de trabajadores aseguran que pueden controlar una situación de riesgo que pueda producirse al realizar trabajos en alturas; el 74\% piensa que los riesgos pueden afectar a más personas y el $90 \%$ aseguran que la gravedad del daño es muy alta.

Los resultados de las variables que tienen una correlación con la magnitud del riesgo en trabajos de operación y mantenimiento en alturas en torres de telecomunicaciones son: la variable (B4) la verificación del estado de los equipos que debe realizar cada colaborador previo a ejecutar trabajos en alturas; (C7) la probabilidad de que más personas resulten afectadas por el riesgo, denominado potencial catastrófico, (C8) denominada a gravedad de las consecuencias y (C4) denominada vulnerabilidad personal.

Con estos resultados se considera que las empresas deberían realizar un plan de comunicación para todos los empleados de las empresas proveedoras de servicios de operación y mantenimiento, el cual abarque las tres variables que permitirán reducir los accidentes de trabajo y enfermedades profesionales al realizar trabajos en alturas en torres de telecomunicaciones. 
Así mismo, las empresas proveedoras de servicios de operación y mantenimiento de torres de telecomunicaciones deben reforzar en sus equipos de trabajo la importancia del uso de equipos de protección personal, aunque la tarea a ejecutar sea de corta duración, así como reforzar el tema que la experiencia no garantiza que no ocurran accidentes de trabajo.

De lo expuesto en la sección de resultados, la parte teórica menciona el Modelo de Creencias de Salud (MCS) de Maiman y Becker, el cual explica la conducta preventiva basada en una gravedad percibida y en una amenaza percibida, esto se comprobó en este estudio al encontrar una correlación entre la magnitud el riesgo y la variable (C8) que se refiere a la gravedad del daño que le puede causar al ejecutar trabajos en alturas en torres de telecomunicaciones y a la correlación con la variable (C3) que hace referencia a cuánto le teme el trabajador al daño que le pueda ocurrir mientras realiza trabajos en alturas.

Por su parte la Teoría de la Acción Razonada (TAR) de Fishbein y Azjen considera la conducta preventiva basada en presiones sociales o en lo que otras personas piensan sobre la conducta preventiva, esto se comprueba en el análisis de la media aritmética de la tabla 7, que demuestra que la variable (B6) "le parece divertido fumar o comer cuando realiza trabajos en alturas", se encuentra en un valor por debajo de dos, lo que indica que a los trabajadores no les parece divertido hacerlo.

Así también la teoría nos muestra que existen investigaciones realizadas acerca de la percepción del riesgo en trabajos en alturas en varias industrias, pero no existe una investigación realizada para trabajos en alturas en empresas de telecomunicaciones, por lo cual esta investigación es una contribución importante incluyendo esta industria que se encuentra en constante crecimiento y en constante cambio.

En lo que se refiere a limitaciones se tiene que a pesar de que el instrumento de investigación aplicado fue de carácter anónimo, existe la posibilidad de que las respuestas tengan un sesgo originado por el temor que pueden tener los trabajadores de ser despedidos debido a que puede observarse un incumplimiento en los procedimientos de trabajo o por informar los errores que tiene la empresa, lo que demostraría una falta de cultura de seguridad y salud en la misma.

Los resultados del estudio no han podido ser comparados con otros similares, ya que no existen hasta el momento información de libre acceso.

\section{Referencias}

Adarraga, P., Hernández, J., Márquez, M., Santacreu, J. (2002). La personalidad en el marco teórico de una teoría del comportamiento humano. Madrid: Pirámide.

Armengou, M., López, E,. (2006). Percepción del riesgo, actitudes y conducta segura de los agentes implicados en los accidentes de trabajo. Retrieved from http://pdfs.wke.es/8/4/9/2/pd0000018492.pdf.

Blanco, A., Caballero, A., Rojas, D., Sanchez, F. (2000). Supuestos teóricos para un modelo psicosocial de las conductas de riesgo”. In: Yubero, S. y Larrañaga E, editor. Sida: Una visión multidisciplinar. España: Universidad Castilla La Mancha.

Chisvert, M., Melià, J., y Pardo. (2001). Un modelo procesual de las atribuciones y actitudes ante los accidentes de trabajo: estrategias de medición e intervención. Revista de Psicología del Trabajo y de las Organizaciones, 17(1), 63-90.

Da Broi, U., Fanzutto, A., Gubiani, R., Moreschi, C., Pergher, G., Secondo, S., \& Veelo, M. (2015). Fatalities Resulting from Falls from Height in Agricultural 
Contexts. Contemporary Engineering Sciences, 8 (25), 1141-1152. doi: $10.12988 /$ ces.2015.56172

Dragoi, G.,Pavalouis, I., Rosu, L., Rosu, S. (2015). Risk assessment of work accidents during the installation and maintenance of telecommunication networks. Environmental Engineering and Management Journal, 14 (9), 2169-2176. doi: 10.30638/eemj.2015.231

Díaz, P. (2015). Prevención de riesgos laborales. Madrid: PARANINFO.

Fischhoff, B., Slovic, P., Lichtenstein, S., Read, S., Combs, B., et al. (1978). How safe is safe enough?: a psychometric study of attitudes towards technological risks and benefits. Policy Sci, 9 (2), 127-152

Galarsi, M., Medina, A., Ledezma, C. y Zanin, L. (2011). Comportamiento, historia y evolución. Fundamentos en Humanidades, XII, 89-123. Retrieved from http://www.redalyc.org/articulo.oa?id=18426920003

González, Y. (2015). Evaluación de la percepción del riesgo ocupacional en trabajadores de una empresa del sector de la construcción en Bogotá D.C. Nova, 13 (23), 93-107. Retrieved from http://www.scielo.org.co/pdf/nova/v13n23/v13n23a09.pdf

Hernández Sampieri, R., Fernández Collado, C., \& Baptista Lucio, P. (2014). Metodología de la investigación. México: McGraw Hill.

Lozano, S., Mazenett, J. (2012). Determinación riesgo ocupacional en población laboral con trabajo en altura por medición sérica de marcadores. Revista de la Facultad de Ciencias de la Salud, 9(1), 23-32. Retrieved from http://www.redalyc.org/articulo.oa?id=512156307004

Macía, F.,Varela, L., Vera, A. (2010). El Estudio de la Percepción del Riesgo y Salud Ocupacional: Una Mirada desde los Paradigmas de Riesgo. Ciencia \& Trabajo, 35(12), 243-250. Retrieved from https://dialnet.unirioja.es/servlet/articulo? codigo $=3218520$

Martínez, J., Morillejo, E., \& Pozo, C. (2002). Percepción de riesgo: Una aproximación psicosocial al ámbito laboral. Prevención, trabajo y salud: Revista del Instituto Nacional de Seguridad e Higiene en el Trabajo, 18 (1), 16-20. Retrieved from https://www.insst.es/InshtWeb/Contenidos/Documentacion/TextosOnline/Rev_I NSHT/2002/18/seccionTecTextCompl2.pdf

Puy, A. (1994). Percepción social del riesgo: Dimensiones de evaluación y predicción. Madrid: Universidad Complutense de Madrid.

Puyal, E. (2001). La conducta humana frente a los riesgos laborales. Determinantes $\begin{array}{llll}\text { individuales } & \mathrm{y} & \text { grupales. } & \text { Retrieved }\end{array}$ https://papiro.unizar.es/ojs/index.php/ais/article/view/199/193.

Salvador, M. Caída de altura: estudio en el cadáver de los diferentes cuadros de fracturas óseas y los factores que intervienen en su producción. Importancia de la evaluación radioscópica. Valencia: Universitat de Valencia

Torres, F. (2017). Determinación de conductas inseguras en conductores de bus y su relación de accidentes de tránsito. Estudio de caso de una empresa de transporte público en Colombia. DYNA, 84 (203), 263-272. doi: 10.15446/dyna.v84n203.67544 
Fecha de recepción: 09/08/2019

Fecha de revisión: 02/09/2019

Fecha de aceptación: 07/12/2019 